\title{
ANDES

www.scielo.cl

\section{Terapia mínimamente invasiva para el manejo de un hematoma subcapsular hepático en un recién nacido}

\author{
Minimally invasive therapy for the resolution of a subcapsular hepatic hematoma \\ in a newborn
}

\author{
aBecado de Cirugía Pediátrica. Hospital Padre Hurtado, Clínica Alemana de Santiago. Santiago, Chile \\ bFacultad de Medicina Clínica Alemana-Universidad del Desarrollo. Santiago, Chile \\ 'Clínica Alemana Santiago. Santiago, Chile \\ ¿Becado de Radiología, Hospital Padre Hurtado, Clínica Alemana de Santiago. Santiago, Chile
}

María Santos C. a,b, Gian Paolo Zamboni T. ${ }^{c}$, Daniel Hasson ${ }^{\mathrm{b}, \mathrm{d}}$, Marina Poblete S. ${ }^{\mathrm{c}}$, Patricio Vargas H.

Recibido: 30 de abril de 2020; Aceptado: 18 de agosto de 2020

¿Qué se sabe del tema que trata este estudio?

Los hematomas hepáticos son una de las complicaciones de la inserción de catéteres umbilicales en recién nacidos. En caso de ser intervenidos, actualmente la laparotomía es el método de elección de abordaje.
¿Qué aporta este estudio a lo ya conocido?

La embolización hepática por angiografía es una alternativa innovadora para el manejo de un recién nacido con un hematoma hepático subcapsular. Es un procedimiento con menos comorbilidades y complicaciones que la laparotomía exploradora.

\section{Resumen}

Objetivo: Describir una alternativa innovadora a la laparotomía exploradora en un recién nacido con un hematoma hepático subcapsular secundario a la inserción de un catéter venoso umbilical. Caso Clínico: Paciente de pretérmino con antecedentes de enfermedad de membrana hialina, hipertensión pulmonar y ductus arterioso persistente amplio, que requirió ventilación mecánica y uso de drogas vasoactivas. Se instalaron catéteres umbilicales, fueron controlados con radiografía de abdomen anteroposterior, en la que impresionaron estar adecuadamente posicionados. Evolucionó con mayor requerimiento de drogas vasoactivas, palidez de pared abdominal y distensión abdominal. Bajo ecografía se diagnosticó un hematoma hepático subcapsular contenido, sin signos de sangrado activo, por lo que se decidió conducta expectante. Aumentó requerimiento de drogas vasoactivas y presentó caída de hematocrito. La ecografía de control encontró un hematoma subcapsular de mayor tamaño, abundante liquido perihepático y se confirmó la posición intraparenquimatosa de la punta del catéter umbilical. Se realizó embolización endovascular a través del catéter umbilical con Gelita ${ }^{\circledR}$, logrando oclusión del trayecto capsular. La ecografía de control mostró disminución del hematoma. Conclusiones: El uso de embolización por angiografía no es comúnmente utilizado en urgencias pediátricas.

Palabras clave:

Hematoma;

Hígado;

Angiografía;

Cateterización;

Venas umbilicales;

Recién nacido

Correspondencia:

María Santos C.

mariasantosc@gmail.com 
Es un procedimiento con menos comorbilidades y complicaciones que la laparotomía exploradora, por lo que debería ser una terapia de primera línea en pacientes como el presentado. Las limitaciones para su realización rutinaria son la disponibilidad de pabellón de angiografía y la presencia de un equipo entrenado de radiología intervencionista.

\section{Keywords:}

Hematoma; Liver; Angiography; Catheterization; Umbilical Veins; Newborn

\section{Introducción}

El uso de catéteres umbilicales en recién nacidos es una técnica comúnmente utilizada, con variadas complicaciones. Se estima que sólo un $73 \%$ de los casos son posicionados a un nivel adecuado ${ }^{1}$. Entre las complicaciones descritas se encuentran el mal posicionamiento del catéter, infección, trombosis, perforaciones y complicaciones hepáticas. Dentro de las complicaciones hepáticas, se puede producir perforación, laceración, absceso estéril, hematoma o ascitis ${ }^{2}$. Actualmente, las complicaciones hepáticas graves requieren manejo quirúrgico.

Presentamos un caso clínico de un recién nacido que presenta un hematoma subcapsular secundario a la instalación de un catéter venoso umbilical, el cual es manejado de manera mínimamente invasiva con angiografía e instalación de Gelita ${ }^{\circledR}$, obteniendo un resultado exitoso y una evolución favorable.

El objetivo de este caso clínico es mostrar una alternativa terapéutica menos invasiva para el manejo de estas lesiones.

\section{Caso Clínico}

Paciente recién nacido gemelar I de pretérmino de 32 semanas, adecuado para su edad gestacional, nacido por cesárea en contexto de trabajo de parto prematuro. Presentó enfermedad de membrana hialina, anemia, hipertensión pulmonar y ductus arterioso persistente amplio. Fue tratado con ventilación mecánica de alta frecuencia oscilatoria, surfactante pulmonar, indometacina, fentanilo, hidrocortisona y dopamina debido a presiones arteriales inestables. Al día de vida se introduce un catéter arterial umbilical (CAU) y un catéter venoso umbilical (CVU) para la administración de nutrición parenteral y fármacos. Se realizó radiografía de control anteroposterior post instalación (figura 1), observando catéter arterial a la altura de D8 y catéter venoso que se insinúa en vena cava inferior, ambos en aparente ubicación correcta.

Al quinto día de vida el paciente persistió grave, evolucionó con distensión abdominal asociado a palidez de pared abdominal y a signos de hipoperfusión en el hemiabdomen inferior, con hematocrito de $41 \%$. Se retiró el CAU, se indicó no utilizar catéter venoso y se realizó radiografía de abdomen, en la que no se observaron hallazgos patológicos. La ecografía abdominal evidenció acentuada cantidad de líquido libre intraabdominal con ecos en suspensión (concordante con hemoperitoneo), abundante líquido subcapsular hepático y una lesión focal hepática de aspecto quístico con gas en su interior, en estrecho contacto con el CVU, sugerentes de hematoma hepático intraparequimatoso y subcapsular (figuras 2 y 3 ). Se interpretaron 


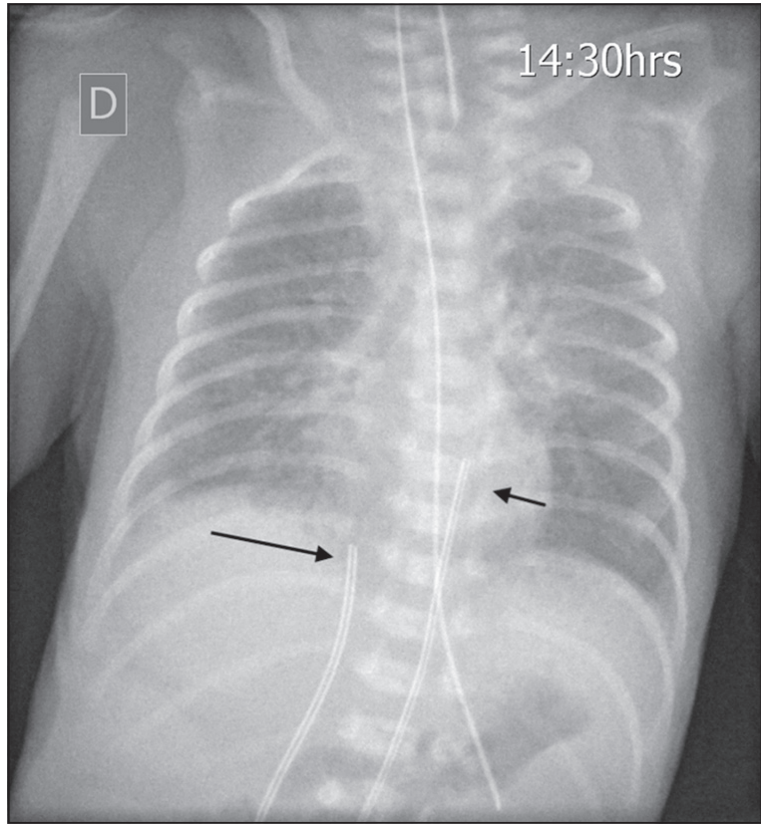

Figura 1. Radiografía de control posinstalación de catéteres umbilicales. Se observa catéter arterial a la altura de D8 (flecha corta) y catéter venoso proyectado en la confluencia del ducto venoso con la vena cava inferior (flecha larga).

estos hallazgos como complicación de malposición de CVU, se decidió mantener una conducta expectante, posponiendo el retiro del CVU (que se mantuvo sin usar) y control ecográfico en $48 \mathrm{~h}$ o antes, en caso de deterioro. Al día siguiente, evolucionó con mejoría de color de pared abdominal, abdomen blando pero labilidad hemodinámica, caída de hematocrito a 29\% (caída de 12 puntos) y mayores requerimientos de drogas vasoactivas. Una nueva ecografía demostró aumento de tamaño del hematoma subcapsular, con el extremo distal del CVU en su interior y líquido libre perihepático de similares características respecto del estudio previo (figura 4). Debido a la mala evolución clínica y los hallazgos imagenológicos, se decidió realizar una angiografía. Se accedió por el catéter umbilical, observándose extravasación de contraste subcapsular, así como también hacia el hematoma intrahepático. Se realizó embolización angiográfica selectiva del hematoma y trayecto intraparenquimatoso a través del mismo catéter con material hemostático absorbible (Gelita $\left.{ }^{\circledR}\right)$, hasta lograr oclusión del trayecto capsular (figura 5). El procedimiento fue realizado sin incidentes, y el CVU fue retirado posteriormente. El paciente evolucionó favorablemente, presentando aumento progresivo de hematocrito a $42 \%$ y disminución progresiva de los requerimientos de drogas vasoactivas. En el control ecográfico 2 meses post intervención, no se pesquisaron complicaciones y el hematoma intraparenquimatoso se observó de menor tamaño, sin evidencias de signos de sangrado reciente.

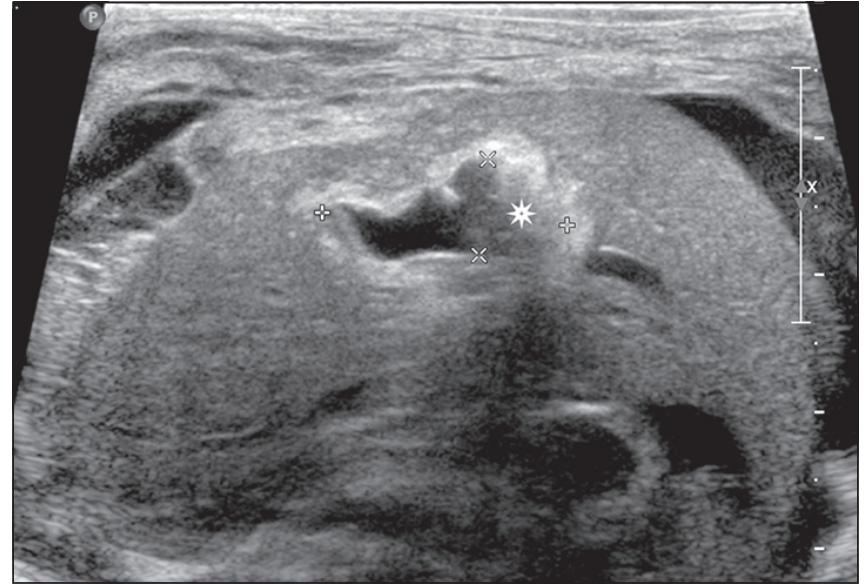

Figura 2. Ecografía abdominal. Imagen quística de bordes anfractuosos, con artefacto de "sombra sucia" en su interior, correspondiente a hematoma intrahepático con aire en su interior.

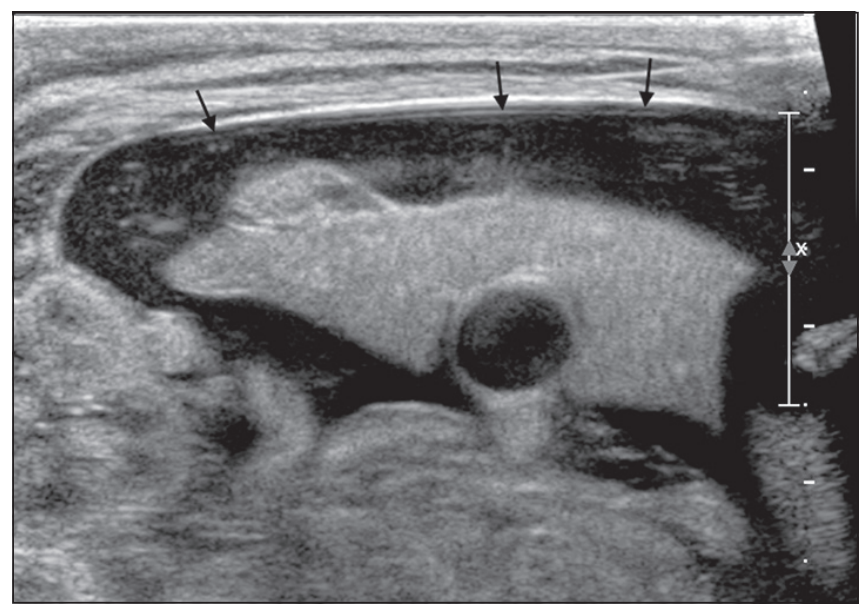

Figura 3. Ecografía abdominal. Extenso hematoma subcapsular hepático (flechas).

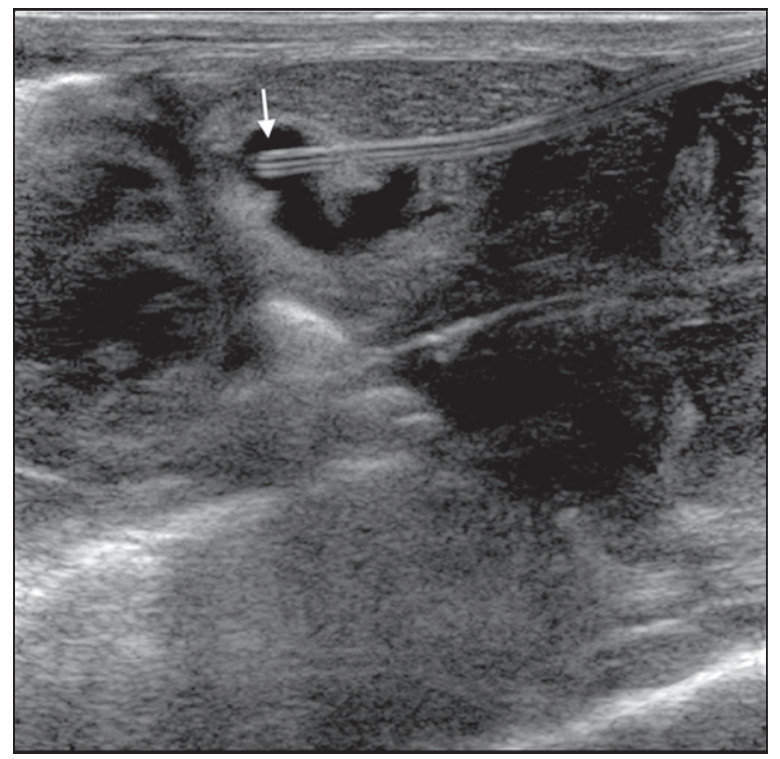

Figura 4. Ecografía abdominal. Punta del catéter en el interior del hematoma intrahepático. 
Figura 5. Imágenes fluoroscópicas durante el procedimiento. Se observa catéter venoso (flecha larga) y sonda de alimentación (flecha corta) (a). Tras la administración de contraste a través del catéter, el hematoma intrahepático se pone de manifiesto (flecha larga) y se identifica además signos de sangrado subcapsular (flecha corta) (b). En la imagen post-embolización se ha retirado el catéter umbilical y se observa material de embolización radioopaco en el trayecto del catéter (flechas cortas) (c).

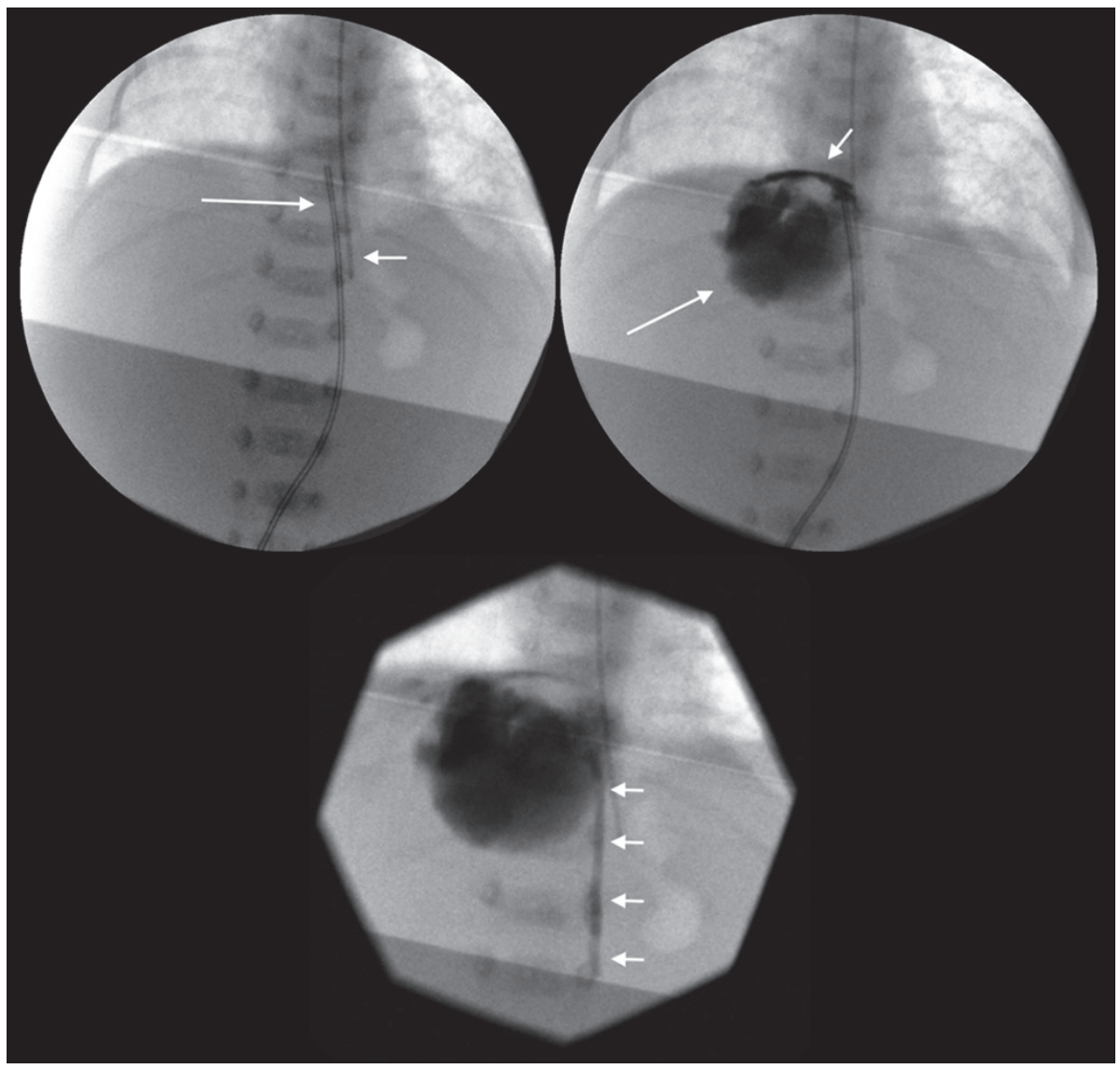

\section{Discusión}

Los hematomas hepáticos son infrecuentes en recién nacidos, sin embargo, están subdiagnosticados, pues generalmente son asintomáticos y en ocasiones son un hallazgo en autopsias ${ }^{3}$. Estos hematomas generalmente son de ubicación subcapsular. Se ha postulado que en recién nacidos de pretérmino existe una mayor fragilidad de la cápsula hepática respecto de los puentes de colágeno que la conectan con el parénquima, lo que sería parte de la patogénesis de estos hematomas ${ }^{4}$. Cuando el hematoma es mayor a $3-4 \mathrm{~cm}$ o sobrepasa la capacidad de ser contenido por la cápsula se hacen sintomáticos. Aumentando el riesgo de generar un hemoperitoneo, el cual puede llevar a un shock hipovolémico con alta mortalidad.

La etiología de los hematomas subcapsulares es variada; siendo en su mayoría traumática, como también secundarios a coagulopatías, hipoxia, sepsis, tumores o malformaciones vasculares congénitas ${ }^{3}$. La compresión de estructuras torácicas y abdominales puede provocar un estiramiento del ligamento coronario insertado en el diafragma produciendo una laceración y hematoma subcapsular, por este motivo el parto vaginal puede ser una causa de hematomas hepáticos.
La sospecha clínica de complicaciones hepáticas esta dada por la presencia de distensión abdominal súbita, asociada a hipotensión y anemia, pudiendo presentarse también con anuria o hipertensión portal aguda $^{2}$. El diagnóstico se confirma con imágenes; el ultrasonido o la resonancia nuclear magnética son la modalidad diagnóstica de elección, dependiendo de la clínica e historia del paciente. La tomografía computarizada también se considera una buena alternativa en caso de no contar con las anteriores ${ }^{5}$.

El manejo de pacientes con hematomas subcapsulares sin alteración hemodinámica ni signos de sangrado activo es conservador; realizando control ecográfico seriado debido al riesgo de rotura, sangrado o infección. En la gran mayoría de los casos se observa resolución espontánea luego de 6-8 semanas. En los casos de pacientes con inestabilidad hemodinámica o síntomas de sangrado activo, es necesario el control vascular. La laparotomía exploradora con packing de la zona sangrante es la medida más comúnmente adoptada, sin embargo, esta técnica es de alto riesgo, asociado a comorbilidades y complicaciones propias del acto quirúrgico. Una opción a esta técnica, es la radiología intervencional que logra embolizar el vaso sangrante.

Considerando los riesgos de una laparotomía en 
pacientes lábiles, el espectro de técnicas mínimamente invasivas se presenta como una alternativa atractiva. El acceso vascular es un aspecto especialmente desafiante en los procedimientos intervencionistas vasculares pediátricos. La vena umbilical es una opción valiosa para pacientes recién nacidos, este acceso es usado con relativa frecuencia en unidades de neonatología y permanece permeable aproximadamente unos 7 días después del nacimiento ${ }^{6,7}$. Como antecedente de un procedimiento similar, Luetic $y$ cols describen la embolización de un hemangioma congénito rápidamente involutivo (RICH) a través de venas umbilicales, si bien en ese caso la vía se utilizó para acceder al territorio arterial a través de un foramen oval patente ${ }^{8}$. Es necesario ponderar los riesgos propios de estos procedimientos en pacientes pediátricos, procurando minimizar la dosis de radiación y vigilar la aparición de complicaciones, las cuales son esencialmente vasculares (hematomas, disección, trombosis y pseudoaneurismas) ${ }^{6}$.

Las imágenes utilizadas para comprobar una adecuada posición de los catéteres son el ultrasonido o la radiografía anteroposterior y lateral. La radiografía de abdomen es actualmente el método más utilizado para el control imagenológico luego de la inserción de un catéter, pero según Michel et al. el ultrasonido es superior a la radiografía para ubicar la punta del catéter, como también ofrece mejor visualización en relación a estructuras vasculares ${ }^{9}$. Derinkuyu et al. también recomiendan el control estricto de la inserción de catéter con ultrasonido ${ }^{5}$. Este caso pone de manifiesto su utilidad para el control post inserción de catéter, como también frente a signos de complicaciones.

En este caso describimos una técnica alternativa para el manejo de estos pacientes, al intervenir de manera mínimamente invasiva, realizando una angiografía umbilical hasta encontrar el sitio de sangrado, para luego introducir material hemostático reabsorbible por el catéter, logrando una adecuada hemostasia. Posterior al procedimiento se debiera seguir al pacien- te con control ecográfico para asegurar la ausencia de sangrado y resolución del hematoma.

\section{Conclusión}

El uso de técnicas mínimamente invasivas guiadas por imágenes ha sido ampliamente utilizado en adultos y se han introducido progresivamente en pediatría durante los últimos años. La embolización endovascular del sitio de sangrado causante del hematoma subcapsular emerge como una alternativa factible y menos invasiva respecto de las técnicas quirúrgicas habituales en esta grave complicación. Es importante destacar, en especial para la realidad chilena, que la menor disponibilidad de pabellón de angiografía y de equipos entrenados de radiología intervencionista es una limitante para hacer mas común este procedimiento. Es una alternativa de terapia, pero no debe retrasar la indicación quirúrgica si los medios no están disponibles.

\section{Responsabilidades Éticas}

Protección de personas y animales: Los autores declaran que los procedimientos seguidos se conformaron a las normas éticas del comité de experimentación humana responsable y de acuerdo con la Asociación Médica Mundial y la Declaración de Helsinki.

Confidencialidad de los datos: Los autores declaran que han seguido los protocolos de su centro de trabajo sobre la publicación de datos de pacientes.

Derecho a la privacidad y consentimiento informado: Los autores han obtenido el consentimiento informado de los pacientes y/o sujetos referidos en el artículo. Este documento obra en poder del autor de correspondencia.

\section{Conflicto de intereses}

Los autores declaran no tener conflicto de intereses.

\section{Referencias}

1. Haase R, Hein M, Thäle V, Vilser C, Merkel N. Umbilical venous cathetersanalysis of malpositioning over a 10 -year period [en alemán]. Z Geburtshilfe Neonatol 2011;215:18-22.

2. Grizelj R, Vukovic J, Bojanic K, et al. Severe Liver injury while Using Umbilical Venous Catheter: Case Series and Literature Review Am J Perinatol. 2014;31:965-74.

3. Costa S, De Carolis M, Savarese I, Lacerenza S, Romagnoli C. Hepatic Hematoma in a Neonate With a High Level of Alpha-Fetoprotein. Eur J Pediatr.
2008;167:591-3.

4. Singer DB, Neave C, Oyer CE, Pinar H. Hepatic subcapsular hematomas in fetuses and neonatal infants. Pediatr Dev Pathol. 1999;2:215-20.

5. Derinkuyu BE, Boyunaga OL, Damar C, et al. Hepatic complications of umbilical venous catheters in the neonatal period. J Ultrasound Med. 2018;37:1335-44.

6. Heran MK, Marshalleck F, Temple $\mathrm{M}$, et al. Joint quality improvement guidelines for pediatric arterial access and arteriography: from the Societies of Interventional Radiology and Pediatric Radiology. Pediatr Radiol. 2010;40:23750.
7. Álvaro RE, Rigatto H. Cardiorespiratory adjustments at birth. En: Avery's Neonatology Pathophysiology \& Management of the Newborn. 6ta ed. Philadelphia: Lippincott Williams \& Wilkins, 2005:285-303.

8. Luetić T, Radoš M, Vuković J, et al. Embolization of a Rapidly Involuting Congenital Hepatic Hemangioma via the Umbilical Vein in a Neonate. J Vasc Interv Radiol. 2019;30:1625-8.

9. Michel F, Brevaut-Malaty V, Pasquali $\mathrm{R}$, et al. Comparison of ultrasound and $\mathrm{X}$-ray in determining the position of umbilical venous catheters. Resuscitation 2012;83:705-9. 